\title{
Contextualizing Child Malaria Diagnosis and Treatment Practices at an Outpatient Clinic in Southwest Nigeria: A Qualitative Study
}

\author{
Juliet Iwelunmor, ${ }^{1}$ Collins O. Airhihenbuwa, ${ }^{2}$ Gary King, ${ }^{2}$ and Ayoade Adedokun ${ }^{3}$ \\ ${ }^{1}$ Department of Kinesiology and Community Health, University of Illinois, Urbana-Champaign, \\ 123 Huff Hall, 1206 South Fourth Street Champaign, IL 61820, USA \\ ${ }^{2}$ Department of Biobehavioral Health, The Pennsylvania State University, 219 Biobehavioral Health Building, \\ University Park, PA 16802, USA \\ ${ }^{3}$ Department of Family Medicine, Lagos State University Teaching Hospital, Lagos 100001, Nigeria
}

Correspondence should be addressed to Juliet Iwelunmor; jiwez@illinois.edu

Received 26 June 2013; Accepted 7 August 2013

Academic Editors: R. Andersson and K. Couper

Copyright (C) 2013 Juliet Iwelunmor et al. This is an open access article distributed under the Creative Commons Attribution License, which permits unrestricted use, distribution, and reproduction in any medium, provided the original work is properly cited.

\begin{abstract}
Background. This study sought to explore contextual features of an outpatient clinic located in southwest Nigeria that enable and/or discourage effective diagnosis and treatment of child malaria. Methods. We conducted in-depth interviews with mothers of 135 febrile children attending a pediatric outpatient clinic in southwest Nigeria. Also, participant observations and informal discussions with physicians were conducted to examine the potential impact of context on effective child malaria diagnosis and treatment. Results. The findings indicate that availability of drugs and laboratory testing for malaria, affordability of antimalarial drugs, access to the clinic (particularly access to pediatricians), adequacy of the outpatient clinic, and acceptability of services provided at the clinic are key contextual factors that influence effective case management of malaria in children. Conclusion. If the Millennium Development Goal 6 of reversing malaria incidence by 2015 particularly among children is to be achieved, it is necessary to identify the contextual factors that may act as potential barriers to effective diagnosis and treatment practices at clinical settings. Understanding the context in which case management of child malaria occurs can provide insights into the factors that influence mis- and over-diagnosis of malaria in clinical settings.
\end{abstract}

\section{Background}

One of the central goals of malaria control programs is to provide effective diagnosis and treatment of malaria particularly in children less than five years of age. To this end, efforts have been made to encourage caretakers of febrile children to seek prompt diagnosis and treatment at health care settings within 24 hours of illness onset. However, despite these efforts, over the past several years, malaria mis- and over-diagnosis have increased dramatically [1-4]. Although the World Health Organization [5] currently recommends "prompt parasitological confirmation by microscopy or alternatively by rapid diagnostic tests (RDTs) for all patients with suspected malaria before treatment is started" in settings where these tools are available, few qualitative lines of evidence exist about the contextual features of health care clinics that influence effective diagnosis and treatment of malaria. Contextual factors are those features of the health care systems which enable and/or discourage effective case management of child malaria [6]. These factors have important implications for reducing the morbidity and mortality from malaria in children. Ignoring the context in which child malaria diagnosis and treatment practices occur, may impede renewed optimism towards improved malaria control and possibly elimination in many endemic countries.

The importance attributed to contextual factors is also underscored by empirical evidence indicating a need to go beyond presumptive (or clinical) diagnosis of malaria in 
children [7-9]. Presumptive diagnosis based on clinical signs and symptoms has been the primary means of diagnosing and treating malaria in many malaria endemic countries [10-12]. It refers to how the disease is understood in the absence of a laboratory confirmation of blood analysis and the course of treatment to be taken. Indeed, in many malaria endemic countries, malaria microscopy which remains the gold standard for laboratory diagnosis remains inaccessible to patients because of poor laboratory infrastructure and understaffing of technical expertise it requires [11]. Even in settings where microscopy is available, referrals for laboratory test rarely happen because in many instances, aspects of such a test (i.e., drawing blood from patients) may also be performed by a physician (as was the case in this study site). Further, when they do happen, they are time consuming and physicians often mistrust the laboratory results and continue to treat those who test negative with antimalarials [13]. For these reasons, knowledge of the contextual factors that influence effective diagnosis and treatment of malaria in children is important for efforts aimed at halting and reversing the incidence of malaria in endemic countries.

In Nigeria, malaria follows a hyperendemic pattern, with peak transmission occurring during the rainy season period (June-July). Nigeria offers a unique opportunity to study the contextual factors that influence effective case management of child malaria for several reasons. First, a quarter of all malaria cases in the World Health Organization in the African region occur in Nigeria [5]. Also, while evidence of systematic decline in malaria cases has been reported in others parts of Africa, malaria remains a persistent problem in Nigeria [5]. According to the National Malaria Control Program in Nigeria [14], malaria is, by far, one of the most important public health problems, representing about $60 \%$ of outpatient visits to health facilities, $30 \%$ of childhood deaths, and $25 \%$ of death in children under one year. Given the burden of malaria in Nigeria, it is possible that contextual features of health care clinics may act in various ways to enable and/or discourage effective case management of malaria in children. At a time of changes in the burden of malaria, with compelling evidence of dramatic decline in malaria transmission in other parts of sub-Saharan Africa [15], it is important to examine the role context plays in influencing effective diagnosis and treatment of malaria. In this paper, we apply the health access livelihood framework to contextualize child malaria diagnosis and treatment practices at an outpatient clinic in southwest Nigeria.

Theoretical Framework: The Health Access Livelihood Framework. The Health Access Livelihood Framework was developed in response to the need to address access to prompt and effective malaria treatment in rural Tanzania $[16,17]$. It is designed to better align health care resources with people's needs, perceptions, and expectations [17]. It combines issues related to health seeking (why, how, and when individuals seek help for illness) with factors that influence access to health care services (availability, accessibility, etc.) to situate the broader context in which effective case management of illness occurs [17]. It consists of five dimensions: availability, affordability, accessibility, adequacy, and acceptability [17].
While availability addresses issues related to the types of services that exist within a health care setting and whether these services correspond with people's needs and expectations, affordability refers to the costs of the services provided (both direct and indirect costs) such as costs of consultations as well as transportation costs and lost time from work [17]. Accessibility is concerned with the geographic distance between services and homes of the intended users [17]. Adequacy examines whether the organization of the health care settings meets the patient's expectations, and acceptability highlights whether or not the information, explanations, and treatment protocols provided take the patient's expectations or perceptions into account [17]. Although this framework has been used to examine the factors that influence access to malaria treatment in a rural setting with limited resources, in urban settings with access to diagnostic tools such as microscopy or malaria rapid diagnosis test, few qualitative attempts have been made to explore how these services align with caretaker's perceptions and expectations. This framework was used in this study to contextualize how services provided at an outpatient clinic in southwest Nigeria align with caretaker's perceptions and expectations of effective diagnosis and treatment of malaria in children.

\section{Methods}

2.1. Setting. This study was conducted in Lagos, one of the largest urban metropolises located in the southwest region of Nigeria. With an estimated population of 12 million people [18], Lagos is also a state and is one of the most populous states in Nigeria with a sociocultural rainbow of people from diverse indigenous backgrounds. It is located within the rainforest region of southwest Nigeria and there are two climatic seasons in Lagos: the dry season and the wet season. The dry season lasts from November to March while the wet (or rainy) season lasts from April to October, with the highest rainfalls occurring during May through July. Malaria transmission in Lagos is intense particularly during the rainy season. This study took place in the pediatric section of an outpatient clinic located in Ikeja, the capital of Lagos. Three times a week on average, the researchers conducted this study at the clinic during the rainy season of June and July, 2010, to explore the mechanisms that guide child malaria diagnosis and treatment decisions at the clinic.

2.2. Study Design and Participants. In-depth interviews, participant observations, informal discussions, and fieldnotes were used to collect data with a purposive sample of mothers with febrile children attending the outpatient clinic and the physicians providing care. Mothers were sensitized to the study in the outpatient waiting room prior to the commencement of the study, and those who provided verbal and written consent were recruited to participate. A total of 135 mothers with febrile children participated in this study. The age range of the mothers was $20-65$, while the children ranged in age from 3 months to 12 years. The majority of the mothers belonged to the Yoruba (59.1\%) and Igbo (28.1\%) ethnic groups in Nigeria. Ethics approval for this study was granted 
by Penn State and the Lagos State University Teaching Hospital.

Verbal consent was also obtained from each physician observed prior to the commencement of the study. Data collection took place in the consultation rooms at the outpatient clinic after routine consultations of mothers with febrile children by physicians. In-depth interviews with mothers focused on perceptions and treatment seeking practices for child's febrile illness prior to clinic attendance. Specifically, mothers were asked to describe how the illness began, what caused the illness, and whether it was severe. They also described their reasons for bringing the child to the clinic, as well as their expectations of the services provided at the clinic. Participant observation focused on interactions between the physicians and mothers. Specifically, using a checklist, the symptoms described by mothers as well as the diagnosis by physicians were recorded. Clinical logic for malaria diagnosis or diagnosis of nonmalaria cases and treatment decisions by physicians were also recorded. Informal discussions with physicians explored their criteria and decision logic for diagnosing malaria in children, their treatment choices, and the potential for malaria mis- and overdiagnoses at this clinical setting.

2.3. Data Analysis. Transcripts of the in-depth interviews, as well as checklists of participant observations, informal discussions, and fieldnotes were analyzed using the content analysis techniques described by Morse and Field [19]. Using the Health Access Livelihood Framework as a guide, responses from the in-depth interviews, informal discussions, as well as checklists of participant observations, and field notes were organized and categorized into expectations about effective diagnosis or child malaria and the resources that enhance or create barriers with effectively managing child malaria at this outpatient clinic. An audit trail of the researcher's decisions and insights were also summarized. Credibility of the data was maintained through triangulation of the multiple sources of data. Also, the data were read in their entirety several times and repeatedly examined so as to obtain a general sense of the information gathered as well as to categorize the material until saturation was reached, that is, until no new themes emerged.

\section{Results}

As stated earlier, the contextual factors are those features of the health care system which either promote or lessen the ability to effectively manage child malaria. In this study, these factors include the availability of drugs and laboratory testing for malaria, affordability of antimalarial drugs, access to the clinic (particularly access to pediatricians), adequacy of clinic, and acceptability of services provided at the clinic.

3.1. Availability of Drugs and Laboratory Testing. This outpatient clinic is known to provide free antimalarial medication for all children less than 5 years of age diagnosed with malaria. Unfortunately, during the course of this study, access to free antimalarial drugs was problematic as the drugs were not always available at the dispensary. Most mothers remarked that the lack of free anti-malaria drugs at the dispensary was a hindrance to the effective treatment of malaria diagnosed in their children. One mother stated the following.

"I came to this clinic because I thought that they give free antimalarial drugs that were of good quality, but they do not have any and I am not sure if I would trust the ones that they have at the market."

In addition to the lack of free antimalarial drugs, although laboratory testing with microscopy is provided at no cost to children attending this clinic, referrals are rare. While some mothers were of the opinion that "it is better to run tests to know the exact problem causing the child's illness," physicians did not recommend it because of time factor, absence of personnel to perform laboratory tasks, and, finally, delay in receiving lab results. In this setting, giving antimalarial treatments to all children with febrile illness was deemed to be necessary by physicians particularly as malaria transmission is hyperendemic in this region of Nigeria. One physician stated the following.

"If I referred a patient for microscopy, it will take at least 2 days before results are available; by then malaria may have worsened, so it is better to treat immediately due to the volume of patients we see in any given day. Moreover, the microscopy laboratories are small, understaffed, and overworked and they lack the equipment to handle the sheer volume of tests needed by patients."

3.2. Affordability of Antimalarial Drugs. One of the key elements for malaria control in Sub-Saharan Africa is prompt treatment with effective antimalarial drugs. Although major efforts are underway to strengthen and promote appropriate utilization of effective antimalarial drugs, barriers imposed by the cost of the new and expensive artemisinin combination therapies may constrain malaria control efforts in multiple ways. For example, findings from the in-depth interviews indicate that affordability of antimalarial drugs can delay prompt treatment of child malaria as evidenced in the following comments.

"I cannot afford to buy meds that the doctor just prescribed because of the cost. I do not have any job or money to buy it now for my child."

Also, it was not uncommon for some mothers to buy chloroquine (despite its known resistance to malaria in this setting) because it was cheap and affordable when compared to the new/expensive artemisinin combination therapies currently in the market. In improving the affordability of antimalarial drugs, one mother stated that "these drugs need to be provided at subsidized price at this clinic so that even poor people can afford to buy them."

3.3. Access to Clinic and Pediatricians. When mothers were asked to describe the length of time it took to travel from their 
homes to the clinic, $41.5 \%$ stated that it took less than 30 minutes, $43.3 \%$ stated it took between 1-2 hours depending on the traffic, and $14.8 \%$ stated that it took over 3 hours to arrive at the clinic. Some of the mothers said they brought their child to this clinic because it is known to provide "free services to everyone." Access to free clinical services was considered to be important, particularly as it addressed the health needs of the poorest who are often deterred from seeking care at most clinics. Some mothers stated that access to the clinic also guaranteed they would receive the "best decision and treatment" for their child's illness. Another component of the clinic resources that matter with the mothers interviewed was "easy access to pediatricians." Indeed, due to ease of access to pediatricians at the clinic, it was not uncommon for some mothers to bring their children to the clinic within 24 hours of illness onset. Easy access to pediatricians also played a significant role in influencing many mother's decisions to travel long distances and in some cases wait 2-3 hours before being seen by the physician at the clinic with little or no complaints. Indeed, our findings revealed that what is often viewed as healthcare barrier or constraint in some settings (i.e., long travelling distances or long waiting times), although important, was insignificant when considered alongside other defining characteristics such as easy access to pediatricians at the clinic.

3.4. Adequacy of Outpatient Clinic. The outpatient clinic caters to the needs of all people residing in the surrounding areas of the clinic as well as people from throughout the country. Although the hours of operation are from 9 a.m. to 3 p.m., most caretakers and their children arrive as early as 6 a.m. to ensure that they are seen as soon as the clinic opens. No prerequisites (such as formal referral letters) are needed to access the clinic's services. As a result, the clinic is readily accessible to patients from all social classes with varied health problems. The caretakers and children who arrive as early as 6 a.m. begin the task of waiting in an area outside the hospital designated as the outside waiting room. The physicians often arrive a little after 8 , and the clinics begin by 9 a.m. The first points of contact for the caretakers and their children are the nurses, matrons, and orderlies at the clinic. They are all women. These women are in charge of ushering the patients from the outside waiting room to the waiting room inside the clinic. The inside waiting area is located outside the physician's office and patients sit in the order of their arrival time or the nurse's arrangement. When it is their turn, they go into the room and begin to narrate their child's illness. Although there are five rooms inside the clinic, only two are designated for general outpatient consultations, and so on many occasions due to lack of space, two physicians share a consultation room, seeing two separate patients at a time. In the context of providing treatment for malaria, as mentioned earlier, the clinic provides free services for children, including free antimalarial drugs for those diagnosed with malaria and free additional testing with microscopy when necessary. Physicians rarely recommended patients for microscopy as they had large queues of patients waiting for consultations. Their priority was to ensure that all waiting patients were seen by a physician during the scheduled hours of operation. Also, unlike in other countries where nurses perform blood work, such task is performed by physicians in this clinic. This means that physicians often have to weigh the time spent drawing blood of one patient (which translates to seeing 3 to 4 patients) or base diagnosis on observation and mothers explanation and history of illness. One physician aptly stated the following.

"Malaria tests with microscopy are cumbersome and in the long-run malaria rapid diagnostic tests are not available indefinitely in this clinic. Patients are many and yes when they come with a temperature, before we think of anything, we have to think of malaria."

3.5. Acceptability of Health Care Services. With regard to acceptability of the health care services provided at the clinic, mothers were asked whether they were satisfied with their consultations (as related to the quality of services provided by physicians and other healthcare workers at the clinic), what they liked best (comments from satisfied mothers), and what the clinic could do to provide better services (comments from dissatisfied mothers). More than half of the mothers (52.6\%) interviewed stated that the quality of care provided at the clinic was excellent. $36.1 \%$ stated that the services were good, while $10.5 \%$ noted that the care provided at the clinic was average. Among mothers who were satisfied with the care that they received, some were of the opinion that the physicians at the clinic were "helpful" and "attentive to their needs." Dissatisfied mothers cited "lack of additional tests" prior to prescribing medications and the "absence of free antimalarial drugs," as key potential barriers for adequate case management of child malaria at this clinic.

\section{Conclusion}

The aim of this study was to illustrate the ways in which contextual factors of an outpatient clinic in southwest Nigeria influence effective diagnosis and treatment of malaria. As child malaria diagnosis remains a major challenge in many endemic countries, the findings indicate that malaria control strategies should take contextual factors into account as they are critical with the effective case management of malaria in children attending health clinics. This is crucial because the success of malaria control strategies does not depend only on the development of effective drugs or vaccines or improved vector control, but also on knowledge of aspects of the context that promote and/or act as barriers to effective diagnosis and treatment. The findings of this study suggest that availability of antimalarial drugs and laboratory testing services, affordability of services, access to clinic and physicians, adequacy of clinics, and acceptability of services are important in addressing access to effective case management of malaria in children attending an outpatient clinic.

Contextual features of health care clinics are also important particularly with the recent advent of malaria rapid diagnostic tests (RDTs) in malaria endemic regions settings [9-11]. Although malaria RDTs could also be useful with effective diagnosis of child malaria, contextual factors such as 
the availability, affordability, access to, adequacy, and acceptability of RDTs may also constrain physician's practice and impoverish their professional judgment. For example, in rural Burkina Faso, Bisoffi and colleagues [20] found that as many as $85 \%$ RDT negative patients were prescribed antimalarials despite the knowledge that negative RDT results excludes presumptive treatment of malaria. Also, in Zambia, Hamer and colleagues [21] noted that when rapid diagnostic tests were performed and reported as negative, 35\% of patients were still prescribed an antimalarial. Simply put, promising advances in malaria rapid diagnostic tests might be futile if the same vigor is not applied to understanding the contexts in which human behaviors occur [22]. Moreover, as noted by Chandler and colleagues [23], "changing ingrained clinical behaviors (i.e., presumptive diagnosis) may be difficult" if attention is not equally given to the role contextual factors play.

Some potential limitations of this study must be duly acknowledged. There is always the possibility that the physicians may have altered their diagnostic and prescribing behavior to err on the side of diagnosing malaria due to the presence of the research study (e.g., Hawthorne effect). To minimize this effect, efforts were made to not interfere with consultations, allowing physicians to diagnose and prescribe child malaria treatment according to their routine. The findings of this study may also be limited due to selection bias since we did not compare participants recruited at this outpatient clinic with those who sought care at other clinics. One caution about our population is that it is plausible for example for mothers with febrile children in search of answers to their child's illness to amplify the severity and persistent sign and symptoms observed in their children in hope to receive additional testing so as to accurately pinpoint the cause of illness. Future studies with mothers with febrile children outside clinical settings are necessary to determine whether this process occurs. Also, the generalizability of our findings is limited since this outpatient clinic may not be representative of other outpatient clinics in malaria-endemic countries. Further, the constraint of space in which this study was conducted may have contributed to bias in reporting some of the findings of this study. However, such space constraint also enabled observations of mundane actions or events to be recorded, particularly with regard to differences between physicians diagnostic decisions and mothers interpretation of their child's illness.

Study findings have implications for improving effective diagnosis and treatment of child malaria in malaria-endemic countries. If the Millennium Development Goal 6 of reversing malaria incidence by 2015 particularly among children is to be achieved, evidently, it is time to examine the contextual factors that are essential for effective diagnosis and treatment of child malaria among children in clinical settings. The results presented in this paper are timely given the increased interest in factors that influence mis- and over-diagnosis of malaria in clinical setting. However, more research is necessary to assess whether these findings remain valid in different clinical settings (i.e., rural clinics and, private versus government owned clinic) and with different participants (i.e., mothers in community settings).

\section{Conflict of Interests}

The authors declare that they have no conflict of interests.

\section{Authors' Contribution}

Juliet Iwelunmor had the original idea for this study which was refined by Collins O. Airhihenbuwa. Juliet Iwelunmor carried out the study. Collins O. Airhihenbuwa and Ayoade Adedokun were involved throughout in supervising the research and in discussing the findings. Gary King contributed to ongoing discussions and commented on the finished paper.

\section{Acknowledgments}

This study was supported by the National Institutes of Health Eunice Shriver National Institute of Child Health and Human Development Predoctoral Fellowship Award Grant no. 1F31HD061196-01A1 and the Penn State African Research Center. We thank the participants who gave generously of their time and the staff of the clinic where this study was conducted for their support for allowing this study to be carried out. We also thank the following for medical doctors for their support during data collection: Drs. Adelakun, Afadapa, Balogun, Disu, Nwosu, and Oladipo.

\section{References}

[1] M. Amexo, R. Tolhurst, G. Barnish, and I. Bates, "Malaria misdiagnosis: effects on the poor and vulnerable," The Lancet, vol. 364, no. 9448, pp. 1896-1898, 2004.

[2] C. I. R. Chandler, R. Mwangi, H. Mbakilwa, R. Olomi, C. J. M. Whitty, and H. Reyburn, "Malaria overdiagnosis: is patient pressure the problem?" Health Policy and Planning, vol. 23, no. 3, pp. 170-178, 2008.

[3] C. I. R. Chandler, C. Jones, G. Boniface, K. Juma, H. Reyburn, and C. J. M. Whitty, "Guidelines and mindlines: why do clinical staff over-diagnose malaria in Tanzania? A qualitative study," Malaria Journal, vol. 7, article 53, 2008.

[4] J. C. C. Hume, G. Barnish, T. Mangal, L. Armázio, E. Streat, and I. Bates, "Household cost of malaria overdiagnosis in rural Mozambique," Malaria Journal, vol. 7, article 33, 2008.

[5] World Health Organization, World Malaria Report, Geneva, Switzerland, 2009, http://whqlibdoc.who.int/publications/2009/ 9789241563901_eng.PDF.

[6] D. C. Glik, W. B. Ward, A. Gordon, and F. Haba, "Malaria treatment practices among mothers in Guinea," Journal of Health and Social Behavior, vol. 30, no. 4, pp. 421-435, 1989.

[7] A. Björkman and A. Mårtensson, "Risks and benefits of targeted malaria treatment based on rapid diagnostic test results," Clinical Infectious Diseases, vol. 51, no. 5, pp. 512-514, 2010.

[8] T. Endeshaw, T. Gebre, J. Ngondi et al., "Evaluation of light microscopy and rapid diagnostic test for the detection of malaria under operational field conditions: a household survey in Ethiopia," Malaria Journal, vol. 7, article 118, 2008.

[9] D. J. Kyabayinze, C. Asiimwe, D. Nakanjako, J. Nabakooza, H. Counihan, and J. K. Tibenderana, "Use of RDTs to improve malaria diagnosis and fever case management at primary health 
care facilities in Uganda," Malaria Journal, vol. 9, no. 1, article 200, 2010.

[10] V. D’Acremont, C. Lengeler, H. Mshinda, D. Mtasiwa, M. Tanner, and B. Genton, "Time to move from presumptive malaria treatment to laboratory-confirmed diagnosis and treatment in African children with fever," PLoS Medicine, vol. 6, no. 1, article e252, pp. 0004-0006, 2009.

[11] H. Hopkins, L. Bebell, W. Kambale, C. Dokomajilar, P. J. Rosenthal, and G. Dorsey, "Rapid diagnostic tests for malaria at sites of varying transmission intensity in Uganda," Journal of Infectious Diseases, vol. 197, no. 4, pp. 510-518, 2008.

[12] A. O. Talisuna and D. N. Meya, "Diagnosis and treatment of malaria: despite accurate diagnostic tests over-diagnosis and presumptive treatment are common practice," British Medical Journal, vol. 334, no. 7590, pp. 375-376, 2007.

[13] M. E. Rafael, T. Taylor, A. Magill, Y.-W. Lim, F. Girosi, and R. Allan, "Reducing the burden of childhood malaria in Africa: the role of improved," Nature, vol. 444, pp. 39-48, 2006.

[14] FMOH, "National malaria control programme in Nigeria," Annual Report, Federal Ministry of Health, 2005.

[15] W. P. O'Meara, J. N. Mangeni, R. Steketee, and B. Greenwood, "Changes in the burden of malaria in sub-Saharan Africa," The Lancet Infectious Diseases, vol. 10, no. 8, pp. 545-555, 2010.

[16] M. W. Hetzel, N. Iteba, A. Makemba et al., "Understanding and improving access to prompt and effective malaria treatment and care in rural Tanzania: the ACCESS Programme," Malaria Journal, vol. 6, article 83, 2007.

[17] B. Obrist, N. Iteba, C. Lengeler et al., "Access to health care in contexts of livelihood insecurity: a framework for analysis and action," PLoS Medicine, vol. 4, no. 10, pp. 1584-1588, 2007.

[18] B. M. Afolabi, W. R. Brieger, and L. A. Salako, "Management of childhood febrile illness prior to clinic attendance in urban Nigeria," Journal of Health, Population and Nutrition, vol. 22, no. 1, pp. 46-51, 2004.

[19] J. M. Morse and P. A. Field, Qualitative Research Methods: Health Professional, Sage, London, UK, 1995.

[20] Z. Bisoffi, F. Gobbi, A. Angheben, and J. Van Den Ende, "The role of rapid diagnostic tests in managing malaria," PLoS Medicine, vol. 6, no. 4, Article ID e1000063, 2009.

[21] D. H. Hamer, M. Ndhlovu, D. Zurovac et al., "Improved diagnostic testing and malaria treatment practices in Zambia," Journal of the American Medical Association, vol. 297, no. 20, pp. 2227-2231, 2007.

[22] H. A. Mwenesi, "Social science research in malaria prevention, management and control in the last two decades: an overview," Acta Tropica, vol. 95, no. 3, pp. 292-297, 2005.

[23] C. I. R. Chandler, S. Chonya, G. Boniface, K. Juma, H. Reyburn, and C. J. M. Whitty, "The importance of context in malaria diagnosis and treatment decisions-a quantitative analysis of observed clinical encounters in Tanzania," Tropical Medicine and International Health, vol. 13, no. 9, pp. 1131-1142, 2008. 


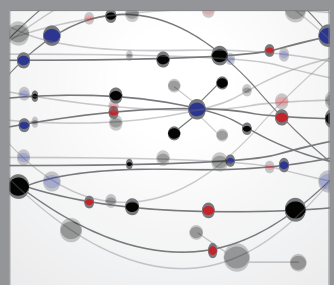

The Scientific World Journal
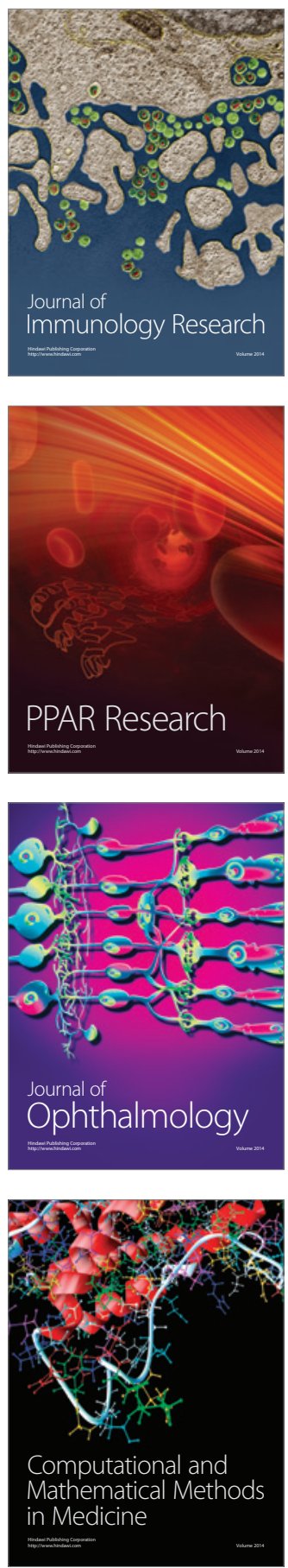

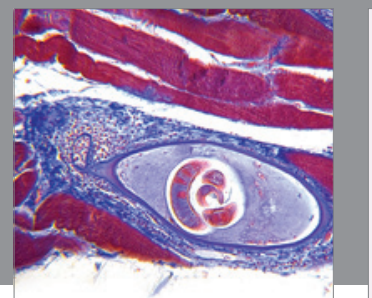

Gastroenterology

Research and Practice
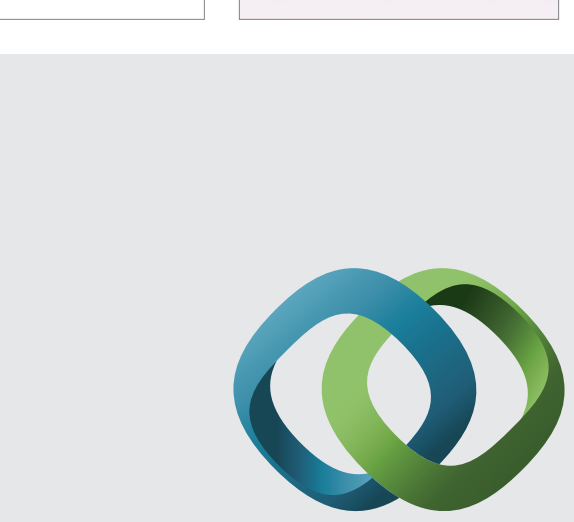

\section{Hindawi}

Submit your manuscripts at

http://www.hindawi.com
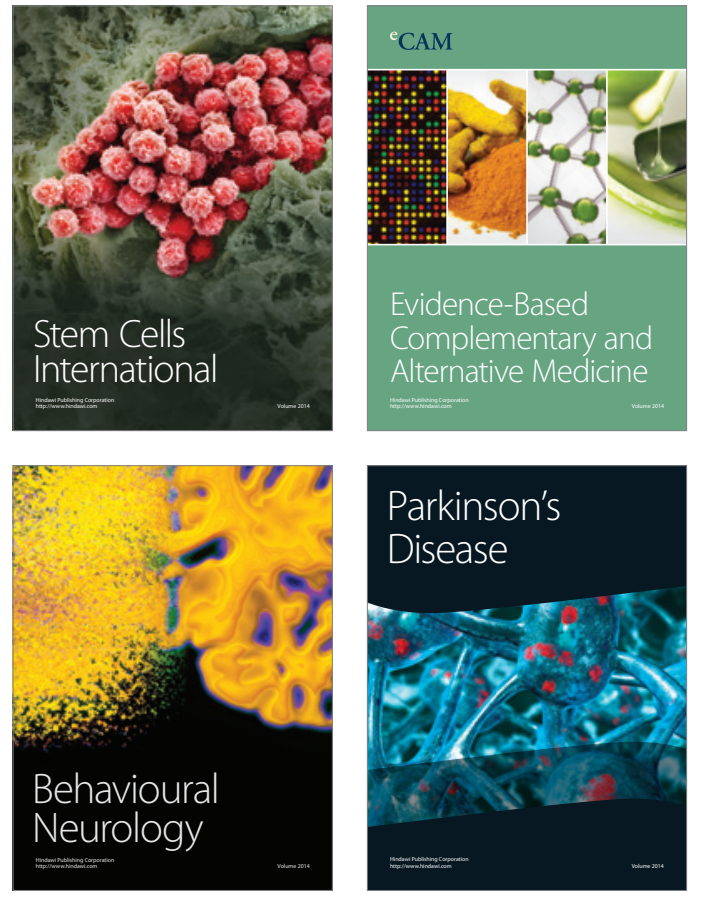
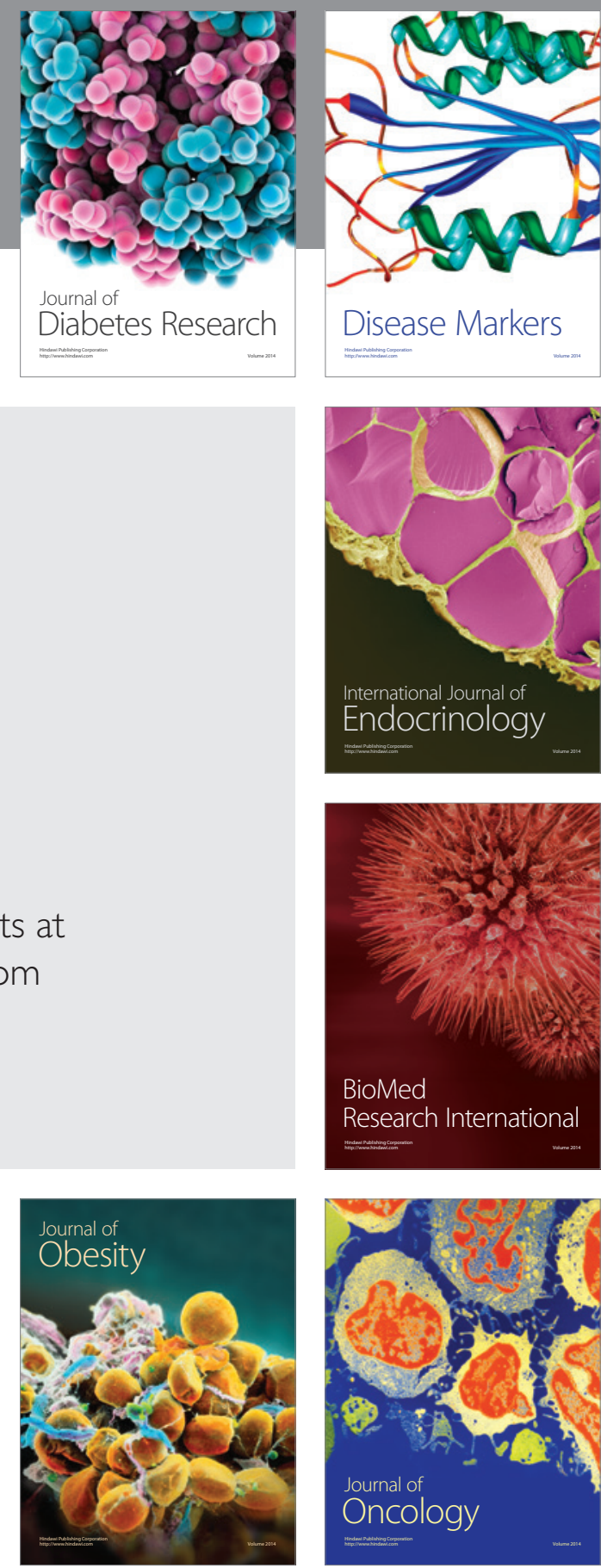

Disease Markers
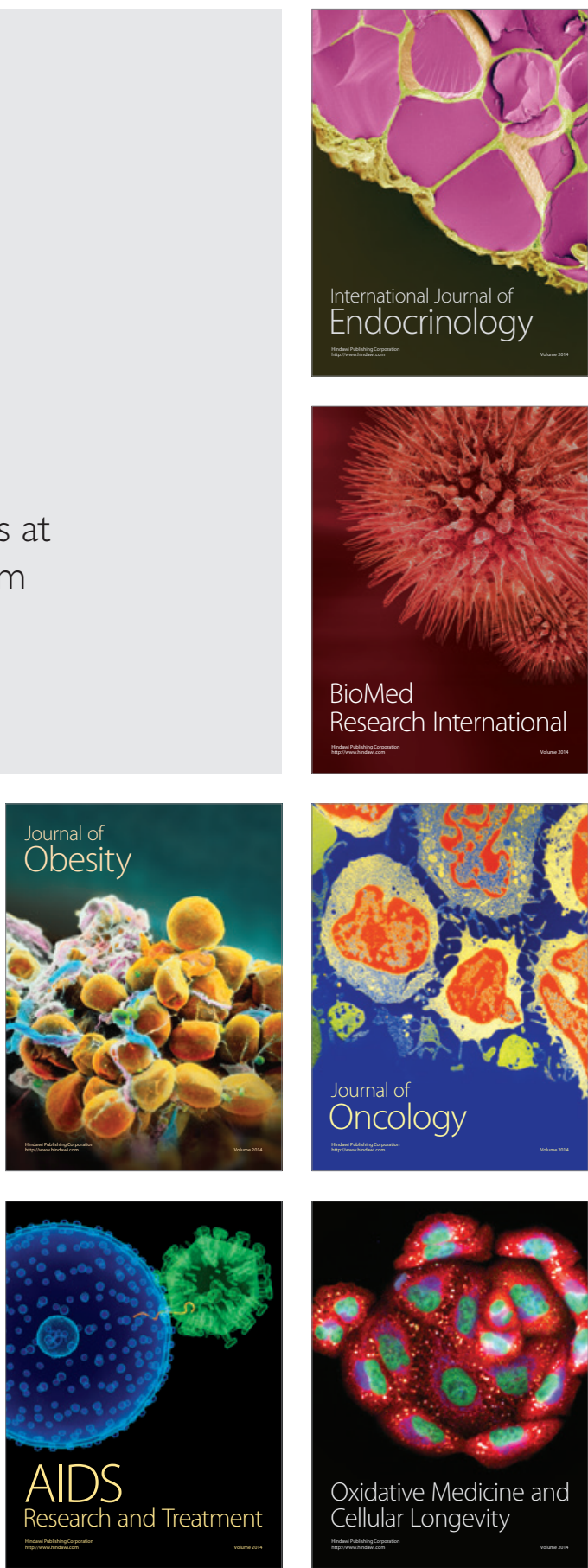